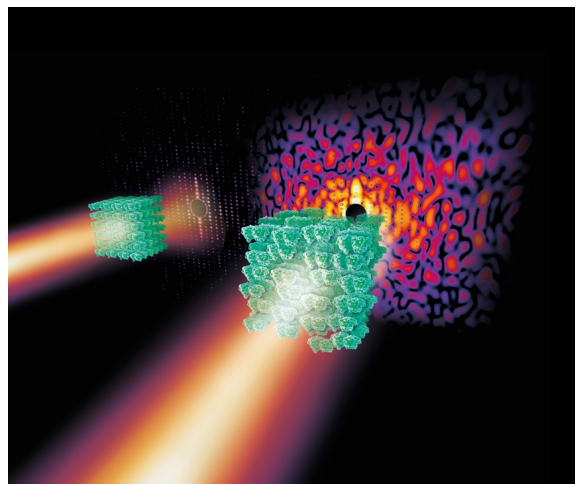

Figure 1. Artistic view of Bragg versus Continous Diffraction from crystals (Credit: DESY, Eberhard Reiman)

Keywords: Free Electron Laser, time-resolved femtosecond crystallography, Photosynthesis, Photosystem II, continous diffraction

\section{KN-13 Transition metal oxides: from structural complexity and topotactic oxygen diffusion pathways to energy applications \\ Werner Paulus ${ }^{1}$}

1. Institut Charles Gerhardt, ICGM-UMR 5253 CNRS Université de Montpellier, 34000 Montpellier, France

email: werner.paulus@univ-montp2.fr

Understanding oxygen diffusion in solid oxides at the atomic scale is an important issue for the development of technological devices such as solid oxide fuel cells (SOFC), as well as membrane based air separators, oxygen sensors and catalytic converters to transform e.g. $\mathrm{NO}$ or $\mathrm{CO}$ from exhaust emissions into $\mathrm{N}_{2}$ and $\mathrm{CO}_{2}^{\mathrm{x}}$ [1-2]. For SOFC e.g., the main drawback of long-term endurance is related to high operating temperatures of around $1000-1250{ }^{\circ} \mathrm{C}$, presenting a key challenge in terms of fatigue due to compositional and microstructural degradation of the respective electrode/membrane materials.

Regarding electrodes materials with mixed ionic and electronic transport properties, two structure types are known today to undergo oxygen intercalation reactions at ambient temperature: the Ruddlesden-Popper series, such as the $\mathrm{RE}_{2} \mathrm{MO}_{4+\mathrm{d}}(\mathrm{RE}=\mathrm{La}, \mathrm{Nd}, \mathrm{Pr} ; \mathrm{M}=\mathrm{Cu}, \mathrm{Ni}, \mathrm{Co})$ members, and the layered double perovskites labeled AA' $\mathrm{B}_{2} \mathrm{O}_{5+\mathrm{d}}$, including the brownmillerite structure, such as $\mathrm{SrMO}_{2.5}^{5+\mathrm{d}}(\mathrm{M}=\mathrm{Fe}, \mathrm{Co})$. We recently demonstrated that oxygen mobility at ambient temperature in brownmillerites is intimately related to low energy phonon modes, which constituted the first evidence of a phonon-assisted oxygen diffusion mechanism [3-4].

We discuss here complex oxygen ordering phenomena during oxygen uptake or release reactions in non-stoichiometric oxides with Brownmillerite and $\mathrm{K}_{2} \mathrm{NiF}_{4}$ type frameworks, essentially explored by in situ neutron scattering and synchrotron radiation. Oxygen doping frequently induces the generation of highly correlated systems for both, oxygen ordering but also for charge and orbital ordering, resulting in giant unit cells. We further discuss the interplay of highly correlated oxygen ordering and related changes in the lattice dynamics to better understand low temperature oxygen mobility, activated by a phonon-assisted diffusion mechanisms. Our studies allow to achieve a microscopic understanding of oxygen diffusion mechanism in solid oxides down to ambient temperature, which is equally important for the synthetic chemist to explore new, kinetically stabilized oxides not available by classical solid state methods [5].

1. Paulus W. et al. JACS 130, 47 (2008) 16080

2. Penkala B. et al. Cat Sci \& Tech, 5 (2015) 4839

3. Ceretti M et al. , J MatChem A, 3, 42 (2015) 21140

4. Perrichon A. et al. JPhysChem C119 (2015) 1557

5. Houchati I. et al. Chem. Mater. 24 (2012) 3811 

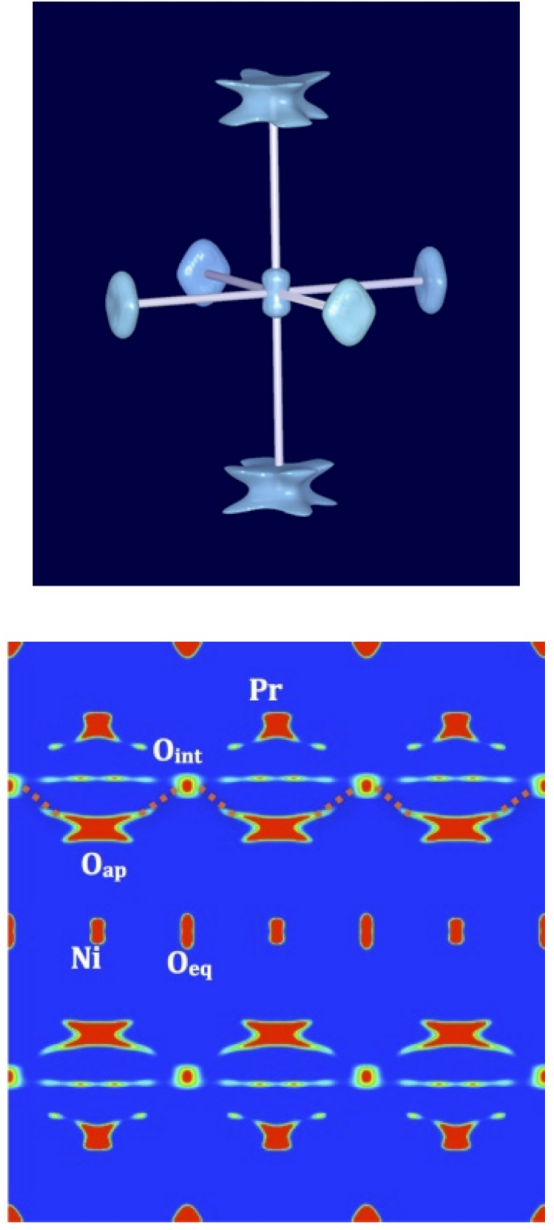

Figure 1. Nuclear density of $\mathrm{Pr}_{2} \mathrm{NiO}_{425} @ 400^{\circ} \mathrm{C}$ from single crystal neutron diffraction and subsequent MaxEnt reconstruction. Top: Anharmonic double potential of the $\mathrm{O}$ atoms of the $\mathrm{NiO}$ octahedra. Bottom: Dynamically activated oxygen diffusion pathway in the $\mathrm{Pr}_{2} \mathrm{O}_{2}$ rock salt layer between $\mathrm{O}_{\text {int }}$ and $\mathrm{O}_{\text {ap }}$ sites[3]

Keywords: transition metal oxides, phonon assisted diffusion, oxygen intercalation, in situ diffraction, inelastic neutron scattering, SOFC

\section{KN-14 Colour X-ray Tomography}

\author{
Bob Cernik ${ }^{1}$
}

1. School of Materials, The University of Manchester, UK

email: B.Cernik@manchester.ac.uk

The twentieth century saw a revolution in the field of $\mathrm{X}$-ray imaging beginning with the acquisition of somewhat rudimentary $2 \mathrm{D}$ radiographs and finishing with the arrival of advanced CT systems capable of producing very high quality $3 \mathrm{D}$ images that can show the internal structure of an object. The most common uses of CT can be found in non-destructive testing; security scanning and in hospitals where modern spiral CT scanners can collect whole body images in minutes. CT works on the basic principle that an X-ray beam incident on an object is absorbed by differing amounts depending upon the composition of the material and the shape of the object. By measuring the transmitted X-rays we obtain 2D radiographs. If we then rotate or translate the sample a number of radiographs can be collected from which 3D, density contrast, images can be mathematically reconstructed. These images can be made sharper by the use of phase or diffraction contrast but are still dominated by absorptive processes. The main limitations of radiography and CT are that they lack insight into the chemical, atomic or crystallographic structure of the object under study. This information can be crucial to fully understanding in-situ chemical processes. Examples of this can be found in corrosion mechanics; alloying behaviour or catalytic reactions. This talk is targeted toward the development and use of bright and dark field colour X-ray imaging systems capable of forming 3D images of relatively large objects using high power, high energy, X-ray sources (from lab and synchrotron). The colour images generated have chemical or physical information in each voxel of the image. Two main approaches will be discussed using high energy monochromatic and white or pink beam radiation. The monochromatic approach is usually called XRD-CT and can be configured to deliver excellent spatial resolution. XRD-CT has recently been demonstrated to work well with PDF data delivering nanocrystalline maps. The energy dispersive methods are faster but have poorer spatial and energy resolution but are more likely to be adopted for non-destructive testing and security scanning applications. EDD imaging has been facilitated by the development of pixelated energy sensitive X-ray detectors and by the development of scattering geometries to improve the signal to noise ratio (SNR) of the weak signals that contain this chemical and structural information.

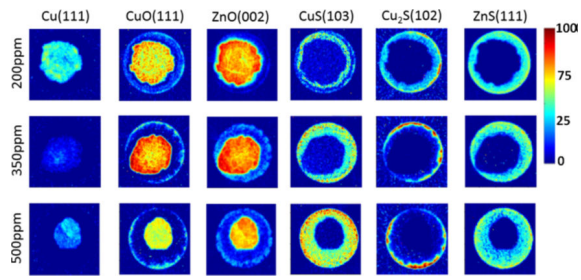

Figure 1. The figure shows how the crystalline phases within a $3 \mathrm{D}$ catalyst body $\left(\mathrm{Cu} / \mathrm{ZnO} / \mathrm{Al}_{2} \mathrm{O}_{3}\right)$ change after various levels of $\mathrm{H}_{2} \mathrm{~S}$ exposure. The 2D reconstructions were based on XRD-CT principles. Beale A $\mathrm{M}$ et al. JOURNAL OF CATALYSIS Vol 314, 94-100 (2014) 